\section{DiAGNÓSTICO DA OTITE MÉDIA AGUDA NA INFÂNCIA}

Método de coleta de evidências: Foram consultadas as bases de dados Medline, através do PUBMED, a base de dados Cochrane de Revisões Sistemáticas e o Registro de Ensaios Controlados da Colaboração Cochrane, através da BVS. A estratégia de busca utilizada baseou-se em perguntas estruturadas na forma de P.I.C.O. (Paciente, Intervenção, Controle, Outcome). A sintaxe de busca resultante foi: ("Otitis Media" [MeSH] AND "Acute Disease"[MeSH] NOT "Mastoiditis" [MeSH] NOT "Otitis Media with Effusion"[MeSH]) AND ("Diagnosis"[MeSH] OR "diagnosis"[Subheading] OR "Diagnostic Techniques, Otological"[MeSH]) AND "Epidemiologic Methods" $[\mathrm{MeSH}]$, recuperando 258 artigos. Foram então acrescidos os limits: all Child: 0- 18 years, clinical trial, resultando em 80 publicações. A seguir foram selecionados pelo abstract 74 trabalhos relacionados diretamente às questões clínicas. Esses trabalhos tiveram a sua força de evidência científica classificada segundo as normas da "Oxford Centre for Evidence Based Medicine". Por fim, selecionaram-se as 13 referências que, pela maior força de evidência científica, consistência e relevância clínica, deram a sustentação às recomendações da presente diretriz.

Graus de recomendação e força de evidência científica:

A: Estudos experimentais ou observacionais de melhor consistência.

B: Estudos experimentais ou observacionais de menor consistência.

C: Relatos de casos (estudos não controlados).

D: Opinião desprovida de avaliação crítica, baseada em consensos, estudos fisiológicos ou modelos animais.

\section{Conflito de interesse}

Nenhum conflito de interesse declarado.

Na otite média aguda (oma), como diferenciar, através dos sintomas e sinais, infecção bacteriana de viral?

Na presença de dor de ouvido, a probabilidade de a criança ter OMA, é 3,0 a 7,3 vezes maior, do que não ter ( $L R+$ : 3,0 a 7,3). Para prevalência de $20 \%$ de OMA, a dor de ouvido confere uma probabilidade pós-teste que varia de 43\% a 65\%. Quando há suspeita dos pais, a probabilidade de a criança ter OMA é 3,4 vezes maior' $(\mathbf{B})$.

Na otite média aguda $(O M A)^{2}(\mathbf{B})$ :

- Os casos de $H$ Influenzae têm sinais e sintomas significativamente mais severos do que os casos com cultura negativa;

- Os casos com cultura positiva têm sinais e sintomas significativamente mais severos do que os casos com cultura negativa;
- O abaulamento e a hiperemia de membrana timpânica (MT) foram os dois sinais mais importantes no diagnóstico diferencial com cultura negativa.

A presença da miringite bolhosa não define etiologia viral. A etiologia da miringite aguda é semelhante à da OMA, em crianças menores de $2 \operatorname{anos}^{3}(\mathbf{B})$.

A probabilidade do diagnóstico de OMA em crianças menores que 4 anos, com infecção de vias aéreas superiores (IVAS), associada a:

- Dor de ouvido é de 7,5 vezes maior;

- Prostração é de 1,3 vezes maior ${ }^{4}(\mathbf{B})$

Em crianças com IVAS o sintoma mais associado com OMA é a dor de ouvido ${ }^{5}(\mathbf{B})$ :

- Com idade < 2anos: risco relativo de 8,5;

- Com idade > 2anos: risco relativo de 47;

- Falso-positivo de I5\%.

Em crianças com OMA menores de 2 anos ${ }^{6}(\mathbf{B})$ :

- A contagem de leucócitos total é significativamente maior nos casos de $S$ Pneumoniae, do que naqueles com cultura negativa ou por $H$ Influenzae;

- A contagem de neutrófilos é significativamente maior nos casos de $S$ Pneumoniae, do que naqueles com cultura negativa ou por $H$ Influenzae.

A probabilidade de etiologia por $S$ Pneumoniae quando na presença de ${ }^{7}(\mathbf{B})$ :

- Alterações severas na MT

- Alterações severas na MT + Temperatura retal $=38^{\circ} \mathrm{C}$

- Dor de ouvido + Temperatura retal (Tret) $=38^{\circ} \mathrm{C}$

5,8 maior:

3,6 maior

- Alterações severas na MT + Tret

$=38^{\circ} \mathrm{C}+$ verão

3,0 maior;

5,0 maior.

A probabilidade de etiologia por $H$ Influenzae quando na presença $\operatorname{de}^{\top}(\mathbf{B})$ :

- Conjuntivite purulenta

- Conjuntivite purulenta + Idade $>$ I ano

9,5 maior;

- Conjuntivite purulenta + História prévia

II maior;

- Sintomas oculares

13 maior;

- Sintomas oculares + História prévia

3, I maior;

4, I maior.

Quais aspectos otoscópicos que sugerem oma? Qual a indicação de timpanometria? Quanto a otoscopia e a timpanometria contribuem com o diagnóstico etiológico?

Na presença de abaulamento da MT a probabilidade da criança ter OMA é $5 \mathrm{I}$ vezes maior do que não ter $(L R+5 \mid)$. As demais razões de verossimilhança nos diversos sinais obtidos na otoscopia são'(B): 
SINAL

Cor da membrana

Opaca
Hemorrágica
Hiperemia

Normal

Posição da membrana

Abaulada

Retraída

Normal

Mobilidade

$\begin{array}{ll}\text { Imóvel } & 31(26-37) \\ \text { Prejudicada } & 4,0(3,4-4,7) \\ \text { Normal } & 0,2(0,19-0,21)\end{array}$

Para prevalência de 20\%, a probabilidade diagnóstica pós-teste, na presença dos diferentes aspectos da membrana timpânica, será'(B):

- Abaulamento da membrana timpânica (LR+5I,0) 94\%;

- Abaulamento + Hemorrágica (LR+ 8,4)

- Abaulamento + Opaca (LR+34,0)

- Abaulamento + Imóvel (LR+ 3l,0)

$8,4(6,7-11)$

$1,4(1,1-1,8)$

$0,2(0,19-0,21)$

$51(36-73)$

$3,5(2,9-4,2)$

$0,5(0,49-0,51)$

- Abaulamento + Coloração ou mobilidade normal (LR- 0,2)

A timpanometria reduz em $20 \%$ a $30 \%$ a quantidade de OMA diagnosticadas ${ }^{8}(\mathbf{B})$.

A timpanometria associada à otoscopia faz diagnóstico de líquido no ouvido médio, com 14\% de falso-positivos ${ }^{9}(\mathbf{B})$.

$\bigcirc$ pico de pressão timpanométrica (PPT) negativo $(<-100)$ em criança menor de 2 anos, sugere ${ }^{10}(\mathbf{B})$ :

- Ausência de: fluído purulento (OR: 0,3) e S. Pneumonia (OR: 0,2);

99\%;

100\%;
- Infecção viral: cultura negativa (OR: 4, I).

A probabilidade de cultura negativa em PPT $<-100$ é 2,8 maior $^{7}(\mathbf{B})$.

Eulália Sakano

LUC L. M. WeckX

Wanderley M. Bernardo

MOACYR SAFFER

Sociedade Brasileira de Pediatria-Departamento de Otorrinolaringologia e Associação Brasileira de Otorrinolaringologia e Cirurgia Cérvico-Facial - Academia de Otorrinolaringologia Pediátrica

Referências

I. Rothman R, Owens T, Simel DL. Does this child have acute otitis media? JAMA 2003; 290: I 633-40.

2. Leibovitz E, Satran R, Piglansky L, Raiz S, Press J, Leiberman A, et al. Can acute otitis media caused by Haemophilus influenzae be distinguished from that caused by Streptococcus pneumoniae? Pediatr Infect Dis J 2003; 22: 509-I 5.

3. Kotikoski MJ, Palmu AA, Nokso-Koivisto J, Kleemola M. Evaluation of the role of respiratory viruses in acute myringitis in children less than two years of age. Pediatr Infect Dis J 2002; 21:636-41.

4. Heikkinen T, Ruuskanen $O$. Signs and symptoms predicting acute otitis media. Arch Pediatr Adolesc Med 1995; 149:26-9.

5. Kontiokari T, Koivunen P, Niemela M, Pokka T, Uhari M. Symptoms of acute otitis media. Pediatr Infect Dis J 1998; 17:676-9.

6. Polachek A, Greenberg D, Lavi-Givon N, Broides A, Leiberman A, Dagan R, et al. Relationship among peripheral leukocyte counts, etiologic agents and clinical manifestations in acute otitis media. Pediatr Infect Dis I 2004; 23:406- 13. 7. Palmu AA, Herva E, Savolainen H, Karma P, Makela PH, Kilpi TM. Association of clinical signs and symptoms with bacterial findings in acute otitis media. Clin Infect Dis 2004; 38: 234-42.

8. Blomgren K, Pitkaranta A. Is it possible to diagnose acute otitis media accurately in primary health care? Fam Pract 2003; 20:524-7.

9. Saeed K, Coglianese CL, McCormick DP, Chonmaitree T. Otoscopic and tympanometric findings in acute otitis media yielding dry tap at tympanocentesis. Pediatr Infect Dis I 2004; 23: I030-4.

10. Palmu A, Syrjanen R, Kilpi T, Pursiainen $H$, Puhakka $H$, Rahko $T$, et al. Negative pressure tympanograms in children less than 2 years of age-different bacterial findings in otitis media by tympanometric results. Int J Pediatr Otorhinolaryngol 2001; 61:61-9. 